\title{
Foundations for Ontology of Persistence: Beyond Talk of Temporal Parts
}

\author{
Fumiaki TOYOSHIMA ${ }^{1}$ \\ GRIIS, Université de Sherbrooke, Sherbrooke, Quebec, Canada
}

\begin{abstract}
Persistence is about how things behave across time. It is generally discussed in terms of endurantism (three-dimensionalism) and perdurantism (fourdimensionalism). Despite the relevance of persistence to ontological modeling, however, there is no clear consensus over how to characterize precisely those two theories of persistence. This paper takes the initial steps towards a foundation for ontology of persistence. In particular, I examine by employing recent findings from philosophy of persistence how some major upper ontologies conceptualize endurantism and perdurantism. My resulting modest suggestion is that formalontological discussion on persistence should be updated by expanding its perspective beyond the topic of whether objects have proper temporal parts or not.
\end{abstract}

Keywords. persistence, temporal part, time, spacetime, upper ontology

\section{Introduction}

Persistence is about how things behave across time. It is usually discussed in terms of two contrasting theories of persistence: endurantism (aka three-dimensionalism) and perdurantism (aka four-dimensionalism). To see the relevance of persistence to ontological modeling, it will suffice to consider its vital role in upper ontologies. An upper ontology (aka foundational ontology), by nature, "deals with general domainindependent categories only" and "has been built and motivated by the upfront and explicit choice of its core principles" [1, p. 3]. A classical example of choices of upper ontologies is an ontological choice [2]: a choice as to whether a certain ontological category or relation is adopted. A relatively widespread ontological choice is the categorical distinction between continuants (objects) and occurrents (events, processes). There is also a growing acknowledgement of meta-ontological choices [3] of upper ontologies: choices that characterize categories and relations foundationally. As de Cesare et al. [3] say, a choice between endurantism and perdurantism can be seen as a meta-ontological choice because it determines the nature of continuants (and occurrents). As a matter of fact, the terms 'endurant' and 'continuant' (resp. 'perdurant' and 'occurrent') are often employed interchangeably in formal ontology.

It is nonetheless controversial what endurantism and perdurantism are supposed to be. As we will see below, they would seem to be characterized differently in different

\footnotetext{
${ }^{1}$ E-mail: toyo.fumming@gmail.com, fumiakit@buffalo.edu. I am indebted to Antony Galton for his highly valuable feedback on this work in its early stage. I appreciate three anonymous reviewers' constructive reviews. I also thank Jean-François Ethier and Christina Khnaisser (in alphabetic order) for some helpful comments on the occasion of the finalization of this paper. Finally, I acknowledge financial support by the SPOR Canadian Data Platform (CIHR).
} 
upper ontologies. Because these two accounts of persistence and a choice between them constitute the crux of ontology of persistence, it will be useful to provide a systematic exploration of hitherto implicit divergent views of persistence in formal ontology.

This paper aims to investigate ontology of persistence from a foundational perspective from which to clarify meta-ontological choices as to persistence. To achieve this goal, I begin by presenting some preliminaries and explaining four existing desiderata for classifying endurantism and perdurantism (Section 2). Then I examine, according to those four criteria, what three major upper ontologies take ontology of persistence to be primarily about: Basic Formal Ontology (BFO), the Descriptive Ontology for Linguistic and Cognitive Engineering (DOLCE), and General Formal Ontology (GFO) (Section 3). Based on this case-study, I consider which standard for characterizing endurantism and perdurantism would be appropriate in formal ontology by employing recent insights from philosophy of persistence; and I also briefly discuss some implications of my argument on persistence for ontology of time (Section 4). Finally, I conclude the paper with a small suggestion on a foundational ontology of persistence and some brief remarks on future work (Section 5).

\section{Ontology of persistence: A general overview}

\subsection{Preliminaries}

The scope of my inquiry will be specified before I present four existing criteria for characterizing endurantism and perdurantism. In the first place, I consider the persistence of continuants, or precisely so-called ordinary material objects: e.g. molecules, people, tables, and planets. Relatedly, I leave aside abstract objects (e.g. sets and numbers) and also "lower-dimensional" objects (e.g. surfaces and boundaries). The term 'object' will be henceforth used in this sense unless otherwise stated. Indeed, one may think that occurrents also persist, as the terms 'occurrent' and 'perdurant' are often used synonymously. However, the usage of the term 'perdurant' is based on a conceptual analogy between occurrents and objects as conceived in the perdurantist's fashion. Thus, persistence is primarily about persistence of continuants (objects) and talk of "persistence of occurrents" would at best convey a perdurantist understanding of objects. ${ }^{2}$ In addition, I take it as the very starting point that an object persists if and only if it exists at one time and also exists at another different time. To take one example, my table persists because it existed yesterday, exists today, and will probably exist tomorrow. I also take for granted that some persisting objects gain and lose parts over time: for instance, my table still continues to exist after one of its edges is chipped off. ${ }^{3}$

Presumably, ontology of persistence is a challenging task for at least two reasons. For one thing, discussion on endurantism and perdurantism (or three- and fourdimensionalism) is generally complicated by significantly different usages of the terms 'endurantism', 'perdurantism', 'three-dimensionalism', and 'four-dimensionalism'.

\footnotetext{
2 One may think that some version of perdurantism (e.g. [4]) would not distinguish between objects and occurrents (not as sharply as endurantism does or can do, at least) by lumping them together under the heading of something four-dimensional. While this might be a proposed view, it would be still important to clarify how the intuitive difference between objects and occurrents could be represented in this framework

3 Therefore, mereological essentialism [5] ("Objects have their parts essentially") is off the table, since it implies that objects cannot gain or lose parts over time.
} 
Insofar as my literature survey is concerned, the latter two terms tend to be used more often than the former two in formal ontology. In philosophy, by contrast, three- (resp. four-) dimensionalism may sometimes mean a specific version of endurantism (resp. perdurantism) or a combination of endurantism (resp. perdurantism) with a particular view of time. For instance, four-dimensionalism is frequently taken, within perdurantism, as the worm theory as compared to the stage theory (see Section 4 for details on these two perdurantist accounts). For the sake of my argumentation, I will employ primarily the terms 'endurantism' and 'perdurantism' to refer loosely to two most general theories of persistence, and only secondarily the terms 'three-dimensionalism' and 'fourdimensionalism' (e.g. in a survey of upper ontologies to be given in Section 3).

For another, persistence is closely intertwined with time, since persistence is in nature about how objects behave across time. However, the fundamental nature of time has been scarcely addressed by prominent upper ontologies [6] or by domain ontologies (see e.g. [7]). To simplify the matter, I make two basic presuppositions on time (but see Section 4 for a brief discussion on persistence under other temporal assumptions). ${ }^{4}$ First, I postulate the classical (non-relativistic) view of (space and) time or spacetime according to which so-called absolute simultaneity holds. ${ }^{5}$ Second, I stipulate the eternalist view of time: the past, the present, and the future exist, or more specifically, past and future times, objects, and occurrents are as real as the present ones [11]. ${ }^{6}$

\subsection{Theories of persistence: Four desiderata}

There are at least four existing desiderata for characterizing the endurantist and perdurantist accounts of persistence $[13,14]$. These criteria may not exhaust all the aspects of preceding discussion on persistence (see e.g. [15]). As we will see in Section 3 , they are still together general and helpful enough to compare some prominent upper ontologies with respect to persistence.

First, one of the most traditional standards for classifying endurantism and perdurantism is (TP) whether objects lack or have proper temporal parts $[16,17]$ :

(E-TP) Objects lack proper temporal parts.

(P-TP) Objects have proper temporal parts. ${ }^{7}$

\footnotetext{
${ }^{4}$ In this paper I remain neutral about the issue of time instants and time intervals, especially of which are ontologically prior to the other (see Galton's [6] detailed discussion, in particular with respect to BFO, DOLCE, and GFO) and also about the debate over time or spacetime between substantivalism and relationalism. As for the former, this might involve the ambiguity of the term 'time' at some points in my explanation (e.g. of the definition of persistence of objects). As for the latter, one may suspect that I am assuming substantivalism because I take for granted the existence of time or spacetime. According to North's [8] reformulation of this substantival/relational dispute, however, both substantivalism and relationalism can be ontologically committed to spatiotemporal structure while disagreeing over whether (facts about) spatiotemporal structure is "grounded" in (facts about) material bodies. Given her proposal, my argument below can be open to both substantivalists and relationalists.

5 See Maudlin's [9] introductory guide for space, time, and spacetime in classical physics and also Bittner's [10] formal ontology of classical physics.

${ }^{6}$ Another motivation behind this assumption than simplicity is the recent argument [12] that eternalism is entailed even by some approaches to quantum gravity (such as loop quantum gravity and string theory) that suggest the non-fundamentality of spacetime, especially of time.

7 The notation ' $\mathrm{E}-\mathrm{X}$ ' (resp. ' $\mathrm{P}-\mathrm{X}$ '), where $X$ is a variable term, means the characterization of $e$ durantism (resp. perdurantism) in terms of $X$.
} 
As for (P-TP), for instance, my table has its "temporal part at a time $t_{l}$ " and its "temporal part at a time $t_{2}$ ", just as it has a flat top and a leg as its spatial parts (see Section 4 for detailed discussion on the definition of temporal parts).

Second, an equally well-known desideratum is (PART) whether objects stand only in time-relative parthood relations [17-19]:

(E-PART) Objects stand only in time-relative parthood relations.

(P-PART) Objects stand in atemporal parthood relations.

To illustrate, the endurantist insists by (E-PART) that the part-whole relation between a leg and my table should be relativized to time such as $t_{l}$. The perdurantist counters by (P-PART) that this leg bears the parthood relation to my table in a timeless way.

Third, (SL) spatiotemporal location (i.e. how objects are located in spacetime) attracts growing attention in contemporary philosophy of persistence $[13,14,20-22]$ :

(E-SL) Objects are three-dimensional.

(P-SL) Objects are four-dimensional.

We will see Donnelly's [13] more rigorous formulation of (SL) based on some auxiliary assumptions about spacetime in Section 4. It should be noted that, as I will illustrate with upper ontologies in Section 3, talk of three- and four-dimensionalism does not ipso facto entail any commitment to (E-SL) and (P-SL), respectively.

Fourth and finally, a comparatively minor criterion is (EXEM) whether objects exemplify properties only in a time-relative way $[14,19]^{8}$ :

(E-EXEM) Objects exemplify properties at a time in virtue of the fact that they exemplify those properties in a way that is relative to the time.

(P-EXEM) Objects exemplify properties at a time in virtue of the fact that their temporal parts at the time exemplify those properties atemporally. ${ }^{9}$

Suppose that my table is white at a time $t_{l}$. This is the case, as the endurantist says by (EEXEM), in virtue of the fact that either my table exemplifies the property of being white that is relativized to $t_{l}$ or my table bears the exemplification relation (that is relativized to $t_{1}$ ) towards the property of being white. Contrariwise, the perdurantist ascribes, based on (P-EXEM), the same state of affairs to the fact that the "temporal part at $t_{l}$ " of my table exemplifies the property of being white in a timeless manner.

\section{Persistence in upper ontologies: A case-study}

I presented above four standards for specifying endurantism and perdurantism: (TP), (PART), (SL), and (EXEM). Given those desiderata, we will look at those formulations of theories of persistence which are provided by three well-known upper ontologies: BFO, DOLCE, and GFO. The focus is upon persistence of objects, but I will sometimes discuss occurrents to know about a perdurantist conception of objects (see Section 2.1).

\footnotetext{
${ }^{8}$ Throughout this paper I use the term 'property' in its most general sense to refer to characteristics of entities, especially of objects.

${ }^{9}$ I present a simplified version of Suzuki's [14] exposition of (EXEM). In particular, (P-EXEM) can be formulated more accurately as follows: “(i) Necessarily, every object has an instantaneous temporal part at every time at which it exists and (ii) objects exemplify properties at a time in virtue of the fact that their temporal parts at the time exemplify those properties atemporally" [14]. Note that the item (i) therein corresponds to (P-TP).
} 


\subsection{Basic Formal Ontology (BFO)}

Basic Formal Ontology (BFO) [23,24] discusses three- and four-dimensionalism in connection with BFO:processes (as a subtype of occurrents) as follows:

One can think of each process as a temporally extended continuum, a spacetime worm (...) this view of spacetime worms is distinct from popular fourdimensionalist views according to which objects (...) would themselves be extended in time and would have temporal parts. BFO does indeed embrace a fourdimensionalist perspective; but it combines this with a three-dimensionalist perspective for continuants (...). [23, p. 124]

BFO would take the core of persistence to be about (SL) ("extended in time") and (TP) ("have temporal parts"). At the same time, BFO accepts (E-SL) and (E-TP) since it subscribes to "a three-dimensionalist perspective for continuants". As evidence of this: "The continuant portion of BFO consists of representations of entities that (1) persist, endure, or continue to exist through time while maintaining their identity, and (2) have no temporal parts" [23, p. 89, emphasis added].

\subsection{The Descriptive Ontology for Linguistic and Cognitive Engineering (DOLCE)}

The Descriptive Ontology for Linguistic and Cognitive Engineering (DOLCE) [2,25] delineates three- and four-dimensionalism as follows:

In general a 3D option claims that objects are: a) extended in a three-dimensional space; b) wholly present at each instant of their life; c) changing entities, in the sense that at different times they can instantiate different properties (indeed, one could say When I was out in the balcony my hands were colder than now). On the contrary a four-dimensional perspective states that objects are: a) space-time worms; b) only partially present at each instant; c) changing entities, in the sense that at different phases they can have different properties (My hands during the time spent out in the balcony, were colder than now). [25, p. 10]

Using Masolo et al.'s [25] notation, a) and c) would correspond approximately to (SL) and (EXEM), respectively. By comparison, b) would be open to interpretation because it is generally contentious what the phrase 'wholly present' (and 'partially present') generally amounts to [26,27]. At least insofar as four criteria under consideration are concerned, however, b) could be also taken to be about (SL) (see also Section 4 for a discussion on a close relationship between (SL) and the expression 'wholly/partially present'). Therefore, I submit that the DOLCE view of persistence is primarily about (SL) and (EXEM) and DOLCE endorses (E-SL) and (E-EXEM), as is shown by the DOLCE category of endurant (continuant). Furthermore, Masolo et al. [25] would seem to describe (PART) as "quite illuminating for our purposes" in discussing the distinction between endurants and perdurants (p. 11, 16). We could therefore recognize the DOLCE commitment to (PART) and (E-PART) as well. 


\subsection{General Formal Ontology (GFO)}

Finally, General Formal Ontology (GFO) [28] discusses persisting objects as follows: "An entity perdures if it persists by having different temporal parts, or stages, at different times, whereas an entity endures if it persists by being wholly present at any time of its existence" [29, p. 180]. GFO would thus seem to think that a general ontology of persistence can be characterized by (TP) ("having different temporal parts, or stages, at different times") and (SL) ("wholly present at any time of its existence").

In striking contrast with BFO and DOLCE, GFO claims that continuants are the creation of the mind and so is their persistence [29,30]. Taken literally, the GFO cognitive view of persistence could imply that there is no persisting object in ontological parlance. It can be also thought however that GFO endorses its original formulation of perdurantism. GFO characterizes continuants in terms of two GFO categories. One is a presential: "an individual which is entirely present at a time-point" [30, p. 309]. The other is GFO:processes, which "have a temporal extension thus cannot be wholly present at a timepoint" [30, p. 310]. GFO asserts principally that, for every continuant Con, there exists a GFO:process $\operatorname{Proc}(\mathrm{Con})$ such that the presentials exhibited by Con equal the GFO:process boundaries of Proc(Con). According to this "principle of object-process integration", the alleged change of continuants is explicable in terms of presentials, which are in turn explainable in terms of GFO:processes. Granted that GFO:processes are "perduring" entities, GFO may well be said to provide a perdurantist account of persistence, as evidenced by Herre's [28,30] claim that GFO is the only four-dimensional upper ontology that is used in applications. ${ }^{10}$ Table 1 briefly summarizes my survey of $\mathrm{BFO}$, DOLCE, and GFO with respect to persistence.

Table 1. A survey of BFO, DOLCE, and GFO upper ontologies with respect to persistence

\begin{tabular}{c|ccc}
\hline Commitment & BFO & DOLCE & GFO \\
\hline w.r.t. (TP) & $\mathrm{X}$ & $\mathrm{X}$ & $\mathrm{X}$ \\
w.r.t. (PART) & $\mathrm{X}$ & $\mathrm{X}$ & \\
w.r.t. (SL) & & $\mathrm{X}$ & \\
w.r.t. (EXEM) & Endurantism & Endurantism & 1) No persisting object \\
w.r.t. its own theory & I.e. (E-TP) and (E-SL) & I.e. (E-PART), (E-SL), & 2) Perdurantism \\
of persistence & & and (E-EXEM)
\end{tabular}

\section{Discussion}

\subsection{Relevance of spatiotemporal location}

According to my survey, BFO, DOLCE, and GFO all share the conviction that (SL) is a suitable standard for characterizing endurantism and perdurantism. To consider this point more carefully, I present Donnelly's [13] formalization of (SL). This requires, first of all, the idea of absolute timeslice. ${ }^{11}$ Assuming that spacetime is a four-dimensional manifold

\footnotetext{
${ }^{10}$ In more detail, Herre [28,30] would seem to employ the term 'three- (resp. four-)dimensionalism' to refer to the thesis that continuants (resp. occurrents) are fundamental to occurrents (resp. continuants). I would prefer to call those two doctrines 'substantialism' and 'processualism', respectively, though [31].

${ }^{11}$ Donnelly [13] assumes Galilean (Neo-Newtonian) spacetime (refer to Maudlin [9] for details). Besides, she claims to presuppose substantivism based on her ontological commitment to spacetime [13, p. 29]. Given
} 
of points that in no way changes or grows, every object is eternally located in this "block spacetime". A spacetime region can be then identified with a non-empty set of spacetime points. Given classical physics, an absolute timeslice is a region which is a maximal set of pairwise (absolutely) simultaneous spacetime points. For the sake of simplicity, we can think of absolute timeslices as time instants (rather than time intervals) in our common understanding of time.

Donnelly also introduces a primitive relation between an object and a spacetime regions: (exact) occupation or (exact) location. Intuitively, an object exactly occupies a spacetime region just in case the object has precisely the same shape, size, and position as the spacetime region does. Roughly speaking, (E-SL) says that a persisting object exactly occupies multiple three-dimensional spacetime regions. (P-SL) says, by contrast, that a persisting object exactly occupies a single four-dimensional spacetime region. Let it be that a spacetime region $r_{l}$ is included in a spacetime region $r_{2}$ if and only if is $r_{l}$ a subset of $r_{2}{ }^{12}$ Donnelly [13, p. 31] characterizes (E-SL) and (P-SL) as follows:

(E-SL) Each persisting object $x$ exactly occupies multiple three-dimensional regions $r_{x t}$ where: (i) each $r_{x t}$ is included in some time and (ii) for each time $t_{0}$ through which $x$ persists, exactly one of $r_{x t}$ is included in $t_{0}$. If there are any nonpersisting objects, each of these objects exactly occupies just one region and this region lies within a single timeslice.

(P-SL) Each persisting object $x$ exactly occupies a unique four-dimensional regions $r_{x}$ which spans $x$ 's entire life (in particular, $r_{x}$ crosses each time through which $x$ persists). If there are any non-persisting objects, each of these objects exactly occupies just one region and this region lies within a single timeslice.

I argue, in line with philosophy of persistence and upper ontologies, that (SL) is an appropriate desideratum for understanding persisting objects (if not fully; see Section 4.3). Characteristically, as Donnelly [13] says, (E-SL) (resp. (P-SL)) might mesh with the endurantist's (resp. perdurantist's) truism that a persisting object is "wholly present" (resp. "partially present") at different times (at which it exists). For, (E-SL) means that a persisting object exactly occupies different (temporally unextended) spacetime regions lying within different timeslices, while (P-SL) means that a persisting object exactly occupies no spacetime region that is "wholly contained" in any timeslice.

\subsubsection{Spatiotemporal location in $B F O$}

Donnelly's [13] formulation of (SL) would help to deepen our understanding of (SL) in the three upper ontologies above discussed. First of all, BFO as such would seem to be silent on the relationship between objects and spacetime regions. BFO indeed has the category of spatiotemporal region [23, pp. 123-124]. However, it is based on the BFO theory of the dynamism of reality according to which a purely spatial ("SNAP") view of the world should be joint together with a purely spatiotemporal ("SPAN") view of the world [32]. The SNAP and SPAN perspectives on the world represent a series of instantaneous snapshots of reality and changes within time spans in reality, respectively.

North's [8] reconstruction of the substantival/relational debate (see Footnote 4), however, Donnelly's proposal may be taken to be neutral as to whether substantivalism or relationalism is adopted.

12 One may prefer to take the inclusion relation between spacetime regions to be primitive by denying Donnelly's [13] identification of spacetime regions as a non-empty set of points. In this sense, her version of (SL) may not be universally accepted, although it would be arguably one of the most explicit formulations of (SL). See also Section 4.1.3 for another limitation. 
In BFO, this SNAP/SPAN divide is well reflected in its top-level categorical distinction between continuants and occurrents. ${ }^{13}$

Consequently, a BFO:spatiotemporal region is "an occurrent entity at or in which occurrent entities can be located" [23, p. 123]. ${ }^{14}$ It is, so to speak, a container within which BFO:processes unfold in the SPAN realm, just as space is a container within which objects and their properties exist in the SNAP realm. Thus, BFO introduces the occupies_spatiotemporal_region relation which has as domain a BFO:process or a BFO:process boundary (i.e. an instantaneous temporal boundary of a BFO:process) and as range a BFO:spatiotemporal region. ${ }^{15}$ This relation cannot be directly used to formalize a BFO- and (E-SL)-based theory of persistence of objects. It could be nevertheless utilized to constrain formally the here introduced "(exact) occupation relation" (OCU) between an BFO:object and a BFO:spatiotemporal region as follows:

\section{OCU $(\mathrm{x}, \mathrm{y}) \rightarrow \exists \mathrm{z}, \mathrm{t}$ participates_in $(\mathrm{x}, \mathrm{z}, \mathrm{t}) \wedge$ occupies_spatiotemporal_region $(\mathrm{z}, \mathrm{y})$ $\wedge$ temporally_projects_onto $(y, t)^{16}$}

Further inquiry along this line is left for the future, such as careful consideration of the conceptual legitimacy of this relation vis-à-vis the SNAP/SPAN view of reality.

\subsubsection{Spatiotemporal location in DOLCE}

Unlike BFO, DOLCE does not explicitly have the category of spacetime region. It is nonetheless natural to think that the DOLCE specification of spatiotemporal locationality of objects would follow its existing "quality-based" treatment of their spatial and temporal locations: "In our ontology, space and time locations are considered as individual qualities like colors, weights, etc.” [25, p. 18]. The DOLCE theory of qualities introduces several technical terms: '(individual) quality', 'quality type', 'quality space', and 'quale' $[2,25]$. Qualities (e.g. the color of this rose) are property particulars that depend specifically on particular entities. Quality types are "partitioners" of qualities: for instance, the color quality type provides the whole color spectrum. Quality spaces (e.g. the "color space") are "classifiers" of qualities of the same quality type, or more precisely mereological sums of all the DOLCE:quality regions (which are a subtype of DOLCE:abstract entities) related to a certain quality type. Qualia (e.g. a particular shade of red) are "values" that qualities have in virtue of their position within a certain quality space. As DOLCE goes, spatial (resp. temporal) locations are qualities that belong to the space (resp. time) quality type and that have as their qualia DOLCE:space (resp. temporal) regions (which are a subtype of DOLCE:quality regions) in the corresponding quality space, namely in the geometric (resp. temporal) space.

In this direction, DOLCE can analyze spatiotemporal locations as qualities that belong to the "spacetime quality type" and that have as their qualia spacetime regions in the "four-dimensional coordinated quality space", i.e. a mereological sum of all the

${ }^{13}$ While retaining its foremost continuant/occurrent distinction, the latest version of BFO [24] would seem to be somewhat distant from this SNAP/SPAN worldview, though.

14 See Bittner's [10] criticism of the BFO occurrent conception of spacetime regions and also Galton's [6] related critical examination of the BFO category of temporal region.

15 See Axiom (15) found in: https://standards.iso.org/iso-iec/21838/-2/ed-1/en/pdf/spatiotemporal.pdf.

16 For details on the participates_in and temporally_projects_onto relations in BFO, see: https://standards.iso.org/iso-iec/21838/-2/ed-1/en/pdf/participation.pdf and https://standards.iso.org/isoiec/21838/-2/ed-1/en/pdf/spatiotemporal.pdf, respectively. 
spacetime-related quality regions. A worry could nevertheless arise as to how well this approach represents the idea of exact occupation that we currently seek. Certainly, DOLCE aims "to capture the intuitive and cognitive bias underlying commonsense" [2, p. 279] and the modeling of spacetime regions within DOLCE should be understood "from the mesoscopic and conceptual level" [2, p. 280] of reality. ${ }^{17}$ Still, spacetime (as well as space and time) and persistence are so fundamental to upper ontologies in general that the DOLCE quality-based view of spatiotemporal location (and perhaps also of spatial and temporal locations) might merit further consideration.

\subsubsection{Spatiotemporal location in GFO}

We will look finally at GFO. While the GFO theories of time [29] and space [34,35] have been recently formalized, the GFO underlying view of spacetime may hinder us from considering persistence in GFO by means of Donnelly's [13] formulation of (SL). GFO conceptualizes space and time as abstractions of the continuum that can be accessed through introspection, hence "phenomenal space" and "phenomenal time". Notably: "space and time cannot be conflated into a homogeneous, four-dimensional system" [34, p. 55]. This contradicts the temporal assumption on which Donnelly's proposal is based: spacetime is a four-dimensional manifold of points. As GFO says, space and time are nonetheless naturally integrated by the object-process integration principle (see Section 3.3). I will return to the topic of persistence in GFO in Section 4.3.

\subsection{Irrelevance of the mere possession of temporal parts}

Let us turn to the (TP) criterion for persistence, on which BFO and GFO would agree. To this date, (TP) remains a fairly common characterization of theories of persistence in philosophy [36] and in formal ontology [31,37]. There is however a growing recognition that (TP) may be an unsuitable standard for understanding endurantism and perdurantism $[13,14]$. Here I present Suzuki's [14] simple argument for this thesis. ${ }^{18}$ First of all, one of the most widespread definitions of temporal parts is arguably Sider's [17] based on the ternary time-indexed parthood relation ${ }^{19}$ :

$x$ is an instantaneous temporal part of $y$ at time instant $t=_{\text {def. }}$ (1) $x$ exists at, but only at, $t$; (2) $x$ is part of $y$ at $t$; and (3) $x$ overlaps at $t$ everything that is part of $y$ at $t$. [17, p. 59]

For instance, a temporal part of my table when it is white is such that (1) it exists at and only at that time; (2) it is part of my table at that time; and (3) for every part $z$ of my table at that time, there is something that is part of the temporal part at that time and that is part of $z$ at that time. Using this definition of temporal parts, Sider explains perdurantism as follows:

(P-TP) Necessarily, every object has an instantaneous temporal part at every time at which it exists.

17 There may be nonetheless a more nuanced approach to spatiotemporal location in compliance with DOLCE. For a pointer to this line of research, see Brodaric et al.'s [33] DOLCE-based formalization of spatial location that is not necessarily based on its quality-based treatment above explained.

18 Donnelly [13] develops a more complex argument by introducing her notion of "temporal segment".

19 Refer to Donnelly [13] and Olson [19] for other alternative definitions of temporal parts. 
It is rather obscure, by contrast, how to offer a (TP)-based formulation of endurantism, i.e. (E-TP), so that (E-TP) can be reasonably compared to (P-TP). A naïve attempt to provide (E-TP) is to deny (P-TP) as follows:

(E-TP*) Possibly, some objects do not have any instantaneous temporal part at some time at which they exist.

Not unnaturally, (E-TP*) would be explanatorily impotent with respect to an accurate understanding of endurantism. A more promising explanation of (E-TP) would be the following, relatively popular idea concerning endurantism:

(E-TP**) Objects do not have any temporal parts (at any time at which they exist).

(E-TP**) would be nonetheless untenable either, because the endurantist theory of persistence of objects (rather than of the structure of objects) would cohere with the existence of temporal parts of objects. To take Sider's [17, p. 64] example, the endurantist would be ontologically committed to (the possibility of) instantaneous objects (objects that exist only at one time instant), which turn out to be (improper) temporal parts given his definition of temporal parts presented above. Sider [17, pp. 6465] further argues, on some auxiliary assumptions that the endurantist can accept, that persisting objects can have (proper) temporal parts. This means that endurantism can be ontologically committed to temporal parts of persisting objects as well.

Furthermore, it is unclear what perduring objects are like according to (P-TP), as says Suzuki [14]. More specifically, it would be hardly understandable why perdurantism compels objects to have so many instantaneous temporal parts during the whole course of their lives. Briefly, (TP) is inappropriate for specifying the endurantist and perdurantist theories of persistence. As Donnelly [13] points out, (TP) may be about what "short-lived" objects there are, but not about how objects persist.

\subsection{Possible relevance of property exemplification}

\subsubsection{More on perdurantism: The worm theory and the stage theory}

As said, my survey would seem to indicate the apparently contradictory GFO conception of persistence: GFO proposes a kind of perdurantism while offering a cognitive interpretation of persistence. To delve into this point, I introduce two prominent variants of perdurantism (mentioned in Section 2.1): the worm theory and the stage theory. The worm theory holds that a persisting object is a four-dimensional "worm" that stretches out through time and that is constructed out of those temporal parts of the object which are connected in some relevant way [16]. ${ }^{20}$ The stage theory maintains that a persisting object consists of "stages" (which would correspond approximately to the worm theorist's temporal parts) in such a way that the object name refers to different stages

${ }^{20}$ Popular answers to the question of linking temporal parts of perduring objects include spatiotemporal continuity [38] and sortal continuity [39]. Sortals are, broadly speaking, are a kind of linguistic terms (or of concepts) that take numerical modifiers, that is, can be associated with numerical adjectives [40]. For instance, the word 'cat' is a sortal because it is a linguistic term that takes numerical modifiers, as is observed by the fact that we can say "two cats". I myself think, following Williams [41], that they are at best necessary conditions for perdurance and only the right kind of causal connection is sufficient to solve this problem, though [31]. 
located within different times $[17,18]$. In this regard, the stage theorist denies diachronic identity of objects because she thinks that objects do not persist in the literal sense of the term. As she adds, objects are said to persist only in the sense that their different stages at different times stand in "temporal counterpart relations" (which would be likened to the worm theorist's connections between temporal parts).

The GFO view of persistence would be stage-theoretic in the sense of coupling a perdurantist ontological view of persistence with an epistemic stand on persistence. In fact, while BFO [23] focuses mainly on the worm theory and DOLCE [25] briefly mentions the stage theory when they discuss perdurantism, the GFO theory of persistence [29] reflects detailed analysis of the problems with the stage theory. It should not be classified as a stage theory in a full-fledged sense of the term, though. ${ }^{21}$

\subsubsection{Complementing spatiotemporal location with property exemplification}

The stage-theoreticity of the GFO view of persistence may help to explain (at least partially) why (SL) might be by itself an inadequate desideratum for understanding persistence in GFO. It is a prevailing orthodoxy that the stage theory is grouped together with the worm theory under the heading of perdurantism [15]. Suzuki [14] argues however that Donnelly's [13] version of (SL) per se would not classify the stage theory uniquely as perdurantism. (SL) admits of two readings, strict and moderate, depending on the construal of the term '(non-)persisting' therein. On the strict reading, the stage theory entails the non-existence of persisting objects and it is ontologically committed only to non-persisting stages, or instantaneous objects. Therefore, the stage theory can satisfy both (E-SL) and (P-SL) and it is categorized either as endurantism or as perdurantism. On the moderate reading (which Suzuki attributes to Donnelly), the term 'persisting' can accommodate the ordinary intuition that objects (e.g. my table) persist and an ontology of the stage theory comprises persisting objects. According to Donnelly's interpretation of the stage theory, a persisting object may exactly occupy a unique three-dimensional spacetime region. Suppose that my table exists at a time $t_{l}$ and at another time $t_{2}$. Then, its $t_{1}$-stage (resp. its $t_{2}$-stage) is my table: namely, an object that is, intuitively, spatially present at and only at $t_{l}$ (resp. $t_{2}$ ). The stage theory would be thus regarded neither as endurantism nor as perdurantism, because it satisfies neither (E-SL) ("multiple three-dimensional regions") nor (P-SL) ("a unique four-dimensional region"). In either case, (SL) does not categorize the stage theory only as perdurantism. ${ }^{22}$

This observation leads Suzuki [14] to suggest that endurantism and perdurantism should be characterized not only in terms of (SL) but also in terms of (EXEM), so that the stage theory can be analyzed uniquely as perdurantism based on the strict (and ontologically more foundational) reading of (SL). This is because the stage theory meets (P-EXEM), but not (E-EXEM). Assuming that my table is white at a time $t_{l}$, this is the case in virtue of the fact that the $t_{l}$-stage of my table exemplifies the property of being white. By (SL) and (EXEM), the stage theory is perdurantism because it satisfies both (P-SL) and (P-EXEM), whereas it is not endurantism because it meets (E-SL) but not (E-

21 "Let us emphasize that (...) [the GFO] approach [to persistence] differs from the stage theory (...). For example, in stage theory processes are considered as mereological sums of stages, temporally extended entities (...). In contrast, processes in GFO do not have such stages as smallest parts.” [29, p. 185]

22 The same criticism can apply to other interpretations of (SL) than Donnelly's [13]. For instance, Balashov [22] provides a (SL)-based formulation of three theories of persistence which he calls 'endurantism', 'perdurantism', and 'exdurantism'. They correspond to endurantism, the worm theory, and the stage theory in the terminology of this paper. He does not give a unifying perdurantist framework for the latter two accounts. 
EXEM). At present, it is not an established approach to classify theories of persistence according to (SL) and (EXEM). Still, this idea would be useful in clarifying the GFO stage-theoretic view of persistence. Lastly, note that DOLCE would espouse (EXEM) (see Section 3.2) and (EXEM) could be generally formalized using DOLCE-CORE [2]: the kind of modular ontology for upper-level entities that is inspired by DOLCE.

\subsection{Persistence and time}

Since persistence and time are intimately related [15], close scrutiny of ontology of persistence may serve to elucidate formal ontologies with respect to their temporal aspect. A foundational view on time can be generally characterized by theory choices concerning two issues [7]. One is the dispute over what we may call 'NOW' between the A-theory (aka the tensed theory) and B-theory (aka the tenseless theory). ${ }^{23} \mathrm{We}$ (directly) experience only the present time, but not any past or future time. NOW seems to move in one direction and the irreversible movement of NOW appears to be the single most important factor of our experience of the "passage" or "flow" of time. The question is whether NOW, the passage of time, and the distinction between the past ("before NOW"), the present ("contemporaneous with NOW"), and the future ("after NOW") are the objective (mind- and language-independent) characteristics of the real world or not. The A-theory says yes: the movement of NOW creates the passage of time from the past through the present towards the future [43]. The B-theory says no: NOW, the passage of time, and the purported past-present-future distinction are nothing more than the features of our experience of time, but not those of fundamental reality of time [11].

The other topic is the controversy over temporal ontology mainly between eternalism (see Section 2.1) and presentism (but see Section 5 for other temporal ontologies). Presentism says that only the present exists, or that only the present times, objects, and occurrents exist [44]. Imagine that one asks: "Does Socrates exist?" and "Does the 5 billion-year-old-earth exist?" The eternalist says yes to both questions, but the presentist says no to them. Because the presentist acknowledges the ontological specialty of the present and argues invariably for the A-theory, I will use the term 'presentism' to refer to a pair of the A-theory with the presentist temporal ontology. In addition, the B-theorist unexceptionally espouses eternalism and their couple is more often than not called 'block universe theory' [17]. While most eternalists are block universe theorists, some eternalists adopt the A-theory and endorse the moving spotlight theory [45]: the view that all the times, objects, and occurrents exist but the presentness is still privileged. A variety of theories of time are briefly summarized in Table 2.

Table 2. Theories of time

\begin{tabular}{c|cc}
\hline & Presentism ("Only the present exists") & Eternalism ("The past, present, and future exist”) \\
\hline A-theory & presentism & moving spotlight theory \\
B-theory & $?$ (unspecified in the literature) & block universe theory
\end{tabular}

The first thing to note is that, contrary to e.g. Merricks [46], endurantism would be compatible with eternalism, at least insofar as either (SL) or (EXEM) is concerned. ${ }^{24}$

23 The terms 'A-theory' and 'B-theory' are usually attributed to McTaggart's [42] terms 'A-series' and 'B-series' of time in his argument for the unreality of time, respectively.

24 By comparison, perdurantism would be hardly consistent with presentism, regardless of whether (SL) or (EXEM) is adopted [14]. I omit to detail this point owing to spatial limitations. 
For one thing, (E-SL) can be generalized enough to be acceptable for both the eternalist and presentist endurantists in such a way that each object exactly occupies a threedimensional spacetime region at each time when it is present. The presentist endurantist would interpret the phrase therein 'at each time when it is present' as saying "whenever it exists", because she thinks that to exist presently is to exist simpliciter [13]. For another, (E-EXEM) is adaptable to eternalism, notably because it can apply to property exemplification regarding diachronic change ("... exemplify properties at a time in virtue of ..." therein; emphasis added) while allowing persisting object to exemplify properties otherwise in the eternalist way that to exist in time is to exist simpliciter [14].

Furthermore, it has been recently argued that, according to (SL), the shift from classical to relativistic spacetime would favor perdurantism (the worm theory, in particular) over endurantism $[13,22,47] .{ }^{25}$ Based on Donnelly's [13] construal of (SL), for instance, (P-SL) can still be efficacious for specifying perdurantism in the relativistic worldview. In contrast, it is unclear how (E-SL) characterizes endurantism under the relativistic assumptions because (E-SL) is deeply rooted in the idea of absolute timeslice, which is incompatible with relativity. This does not ipso facto mean the inconsistency between endurantism and relativistic modern physics, but it is equally true that some substantive work is required for a relativistic reconstruction of (E-SL) $[20,48]{ }^{26}$

All those considerations as to the relationship between persistence and time could help to provide a better understanding of time in upper ontologies [6]. Let me focus only on BFO for space reasons. First of all, BFO adopts a realist approach to ontology development which prescribes that ontologies should represent entities in reality [51]. Since BFO endorses endurantism (see Section 3.1) and endurantism turns out to be compatible with eternalism (as well as the presentist temporal ontology), BFO may be willing to embrace the moving spotlight theory or the block universe theory, rather than presentism. For one thing, eternalism would serve better the general purpose of simpler ontological modeling, given the BFO principle of ontological realism. ${ }^{27}$ Notably, Galton [6] focuses on the BFO category of zero-dimensional temporal region as illustrated by "right now" [23, pp. 124-125]. ${ }^{28}$ He states that, if it is to be taken seriously, this "right now" would entail an ontological commitment to the A-theory, granted the BFO realist methodology. ${ }^{29}$ Following his advice to avoid the potentially problematic A-theoretic property of being "right now", BFO may be further motivated to embrace the B-theory, above all the block universe theory. Finally, it remains to be seen whether and how the BFO endurantist framework can hold in the relativistic setting (see also [10]). and GFO.

${ }^{25}$ See also Galton's [6] detailed discussion on special relativity, especially in relation with BFO, DOLCE,

${ }^{26}$ For persistence under another physical assumption, see Pashby's [49] argument that a (SL)-based characterization of persistence in general (which is not restricted to Donnelly's [13]) can be problematic for both endurantism and perdurantism in quantum mechanics. Additionally, (P-EXEM) may deserve further consideration in quantum mechanics, given his other argument [50] that persisting quantum objects do not have temporal parts.

27 The latest version of BFO [24] elucidates the term 'entity' as "anything that exists or has existed or will exist" (see Section A.1.2.1 of the Excel file named "bfo-2020-terms.xlsx" found in: https://standards.iso.org/iso-iec/21838/-2/ed-1/en/). This might be possibly construed as a commitment to eternalism. In practice, however, realist ontologies would represent entities only in the past and the present, but not in the future, since we are presently never certain about (entities in) the future.

28 The latest version of BFO [24] would seem to drop out this example, though. See Section A.1.2.75 "zero-dimensional temporal region" of the Excel file named "bfo-2020-terms.xlsx" found in: https://standards.iso.org/iso-iec/21838/-2/ed-1/en/.

29 See also Bittner's [10] criticism of the more general BFO category of temporal region. 


\section{Concluding remarks}

This paper aimed at initial elucidation of a foundation for ontology of persistence. For this purpose, I specified four existing desiderata (viz. (TP), (PART), (SL), and (EXEM)) for characterizing endurantism and perdurantism, examined persistence in three upper ontologies (BFO, DOLCE, and GFO) by those criteria, and discussed some key topics emerging from this survey. My overall contention is the modest proposal that formalontological investigation into persistence should be updated by expanding its perspective beyond the issue of whether objects have or lack proper temporal parts. ${ }^{30}$

I conclude with three brief remarks on future work. First, given the relevance of (SL) to persistence, spatiotemporal locationality of objects will require further exploration (see Section 4.1.1 for a pointer to this direction of inquiry in BFO). Second, careful investigation is warranted into the nature of time [6], especially into other temporal ontologies than discussed above: e.g. the growing block theory [52,53] ("The past and the present exist, but the future does not"; see also [7,54]) and the shrinking block theory [55] ("The present and the future exist, but the past does not"). Third, it is well worth considering the relationship between persistence and scientifically important notions of "natural necessity" [56]: e.g. causation, dispositions, laws of nature, and counterfactuals.

\section{References}

[1] Borgo S, Hitzler P. Some open issues after twenty years of formal ontology. In: Proceedings of FOIS2018. Amsterdam: IOS Press; c2018. p. 1-9.

[2] Borgo S, Masolo C. Ontological foundations of DOLCE. In: Poli R, Healy M, Kameas A, editors. Theory and applications of ontology: computer applications. Springer; 2010. p. 279-95.

[3] de Cesare S, Gailly F, Guizzardi G, Lycett M, Partridge C, Pastor O. 4th international workshop on ontologies and conceptual modeling (Onto.Com). In: Proceedings of JOWO2016. CEUR Workshop Proceedings, vol. 1660; c2016. p. 1-3.

[4] Quine WV. Identity, ostension, and hypostasis. J. Philos. 1950 Oct;47(22):621-33.

[5] Chisholm RM. Person and object: a metaphysical study. La Salle (IL): Open Court; 1976. 230 p.

[6] Galton A. The treatment of time in upper ontologies. In: Proceedings of FOIS2018. Amsterdam: IOS Press; c2018. p. 33-46.

[7] Toyoshima F. Ontology of time for the digital humanities: a foundational view. In: Proceedings of JOWO2019. CEUR Workshop Proceedings, vol. 2518; c2019. p. 1-6.

[8] North J. A new approach to the relational-substantival debate. In: Bennett K, Zimmerman DW, editors. Oxford studies in metaphysics, vol. 11. Oxford: Oxford University Press; 2018. p. 3-43.

[9] Maudlin T. Philosophy of physics: space and time. Princeton University Press; 2012. 200 p.

[10] Bittner T. Formal ontology of space, time, and physical entities in classical mechanics. Appl. Ontol. 2018 May;13(2):135-79.

[11] Mellor H. Real time II. London: Routledge; 1998. 160 p.

[12] Le Bihan B. String theory, loop quantum gravity and eternalism. Eur. J. Philos. Sci. 2020 Mar;10:17. https://doi.org/10.1007/s13194-020-0275-3

[13] Donnelly M. Endurantist and perdurantist accounts of persistence. Philos. Stud. 2011 May;154(1):27-51.

[14] Suzuki I. What do four-dimensionalism and three-dimensionalism disagree about? J. Jpn. Assoc. Philos. Sci. 2017;44(1-2):15-33. [in Japanese]

[15] Haslanger S, Kurtz RM, editors. Persistence: contemporary readings. Bradford; 2006. 483 p.

[16] Lewis DK. On the plurality of worlds. Oxford: Blackwell; 1986. 276 p.

[17] Sider T. Four-dimensionalism. Oxford: Oxford University Press; 2001. 288 p.

[18] Hawley K. How things persist. Oxford: Oxford University Press; 2002. 232 p.

${ }^{30}$ I did not discuss (PART) in detail for the sake of space, but see Donnelly's [13] argument (which Suzuki [14] and I myself find plausible) that (PART) is an unsuitable criterion for specifying theories of persistence. It is interesting to note that Merricks [46] would seem to employ (PART) in his argument for the incompatibility between endurantism and eternalism. 
[19] Olson ET. Temporal parts and timeless parthood. Noûs. 2006 Dec;40(4):738-52.

[20] Gilmore C. Where in the relativistic world are we? In: Hawthorne J, editors. Philosophical perspectives, vol. 20, metaphysics. Oxford: Blackwell; 2006. p. 199-236.

[21] Sattig T. The language and reality of time. Oxford: Clarendon Press; 2006. 240 p.

[22] Balashov Y. Persistence and spacetime. Oxford: Oxford University Press; 2010. 240 p.

[23] Arp R, Smith B, Spear AD. Building ontologies with Basic Formal Ontology. MIT Press; 2015. 248 p.

[24] ISO/IEC 21838-2 [internet]. Information technology - Top-level ontologies (TLO) - Part 2: Basic Formal Ontology (BFO); 2020 Mar. Available from: https://www.easybib.com/reference/guide/apa/website

[25] Masolo C, Borgo S, Gangemi A, Guarino N, Oltramari A. Wonderweb deliverable D18 - ontology library (final). LOA-NCR-ISTC; 2003. Available from: http://wonderweb.man.ac.uk/deliverables/D18.shtml

[26] Simons P. Location. Dialectica. 2004 Jun;58(3):341-47.

[27] Crisp TM, Smith DP. 'Wholly present' defined. Philos. Phenomenol. Res. 2005 Sep; 71(2):318-44.

[28] Herre H. General Formal Ontology (GFO): A foundational ontology for conceptual modelling. In: Poli R, Healy M, Kameas A, editors. Theory and applications of ontology: computer applications. Springer; 2010. p. 297-345.

[29] Baumann R, Loebe F, Herre H. Axiomatic theories of the ontology of time in GFO. Appl. Ontol. 2014; 9(3-4):171-215.

[30] Herre H. Persistence, change and the integration of objects and processes in the framework of the General Formal Ontology. In Petrov V, Scarfe AC, editors. Dynamic being: essays in process-relational ontology. Cambridge Scholar Publishing; 2015. p. 337-57.

[31] Toyoshima F. Linking temporal parts in processual biological ontology. In: Proceedings of JOWO2019. CEUR Workshop Proceedings, vol. 2518; c2019. p. 1-8.

[32] Grenon P, Smith B. SNAP and SPAN: towards dynamic spatial ontology. Spat. Cogn. Comput. 2004 Mar;4(1):69-103.

[33] Brodaric B, Hahmann T, Grüninger M. Water features and their parts. Appl. Ontol. 2019 Feb;14(1):1-42.

[34] Baumann R, Loebe F, Herre H. Towards an ontology of space for GFO. In: Proceedings of FOIS2016. Amsterdam: IOS Press; c2016. p. 53-66.

[35] Baumann R, Loebe F, Herre H. Properties defined on the basis of coincidence in GFO-space. In: Proceedings of JOWO2019. CEUR Workshop Proceedings, vol. 2518; c2019. p. 1-10.

[36] Wasserman R. Theories of persistence. Philos. Stud. 2016 Apr;173(1):243-50.

[37] Verdonck M, Sales TP, Gailly F. A comparative illustration of foundational ontologies: BORO and UFO. In: Proceedings of JOWO2018. CEUR Workshop Proceedings, vol. 2205; c2018. p. 1-12.

[38] Hirsch E. The concept of identity. Oxford: Oxford University Press; 1982. 336 p.

[39] Wiggins D. Sameness and substance renewed. Cambridge: Cambridge University Press; 2001. 274 p.

[40] Grandy RE. Sortals. In: Zalta EN, editor. The Stanford encyclopedia of philosophy. Winter 2016 ed. Available from: https://plato.stanford.edu/archives/win2016/entries/sortals/

[41] Williams N. Powerful perdurance: linking parts with powers. In Jacobs JD, editor. Causal Powers. Oxford: Oxford University Press; 2017. p. 139-64.

[42] McTaggart JME. The unreality of time. Mind. 1908 Oct;17(68):457-74.

[43] Prior AN. Thank goodness that's over. Philos. 1959 Jan;34(128):12-17.

[44] Ciuni R, Torrengo G, Miller K, editors. New papers on the present: focus on presentism. Philosophia Verlag; 2013. 340 p.

[45] Cameron RP. The moving spotlight: an essay on time and ontology. Oxford: Oxford University Press, 2015. $240 \mathrm{p}$.

[46] Merricks T. On the incompatibility of enduring and perduring entities. Mind. 1995 Jul;104(415):521-31.

[47] Gilmore C. Persistence and location in relativistic spacetime. Philos. Compass. 2008 Nov;6(3):1224-54.

[48] Gibson I, Pooley O. Relativistic persistence. In: Hawthorne J, editor. Philosophical perspectives, vol. 20, Metaphysics. Oxford: Blackwell; 2006. p. 157-98.

[49] Pashby T. How do things persist? Location relations in physics and the metaphysics of persistence. Dialectica. 2016 Sep;70(3):269-309.

[50] Pashby T. Do quantum objects have temporal parts? Philos. Sci. Dec 2013; 80(5):1137-47.

[51] Smith B, Ceusters W. Ontological realism: A methodology for coordinated evolution of scientific ontologies. Appl. Ontol. 2010 Nov;5(3-4):139-88.

[52] Broad CD. Scientific thought. London: Kegan Paul; 1923. 555 p.

[53] Correia F, Rosenkranz S. Nothing to come: a defence of the growing block theory of time. Springer Verlag; 2018. 197 p.

[54] Galton A. Guarino's possibilism. In Borgo S, Ferrario R, Masolo C, Vieu L, editors. Ontology makes sense: essays in honor of Nicola Guarino. Amsterdam: IOS Press; 2019. p. 167-76.

[55] Casati R, Torrengo G. The not so incredible shrinking future. Analysis. 2011 Apr;71(2):240-4.

[56] Toyoshima F. Natural necessity: an introductory guide for ontologists. Appl. Ontol. 2020 Feb;15(1):6189. 